\title{
Quiste hidatídico renal simulando hipernefroma
}

J.M. Abascal Junquera, S. Esquena Fernández, R. Martos Calvo, C. Ramírez Sevilla, C. Salvador Lacambra, A. Celma Doménech, E. Trilla Herrera, I. De Torres*, J. Morote Robles

Servicios de Urología y Anatomía Patológica*. Hospital General Universitario Vall d'Hebron. Barcelona.

Actas Urol Esp 2005; 29 (2): 223-225

\section{RESUMEN}

\section{QUISTE HIDATÍDICO RENAL SIMULANDO HIPERNEFROMA}

Objetivo: Presentar un nuevo caso de quiste hidatídico renal de diagnóstico iconográfico complejo.

Material Y Métodos: Se describe la clínica, diagnóstico y tratamiento de una masa renal compleja y posterior confirmación histológica tras la exéresis de la misma. Revisión de la literatura.

Conclusiones: la hidatidosis renal es una localización infrecuente de esta patología. Es importante tenerla en cuenta a la hora del diagnóstico diferencial en el contexto de masas renales complejas. Existen procedimientos diagnósticos que nos pueden ayudar a confirmarla ante su sospecha. La cirugía es el tratamiento de elección en la mayoría de los casos.

Palabras clave: Quiste hidatídico renal.

\section{ABSTRACT}

\section{RENAL HYDATIDIC CYST SIMULATING HYPERNEPHROMA}

Objective: To present a new case of hydatid cyst of the kidney with a difficult radiographic diagnosis.

Material And Methods: We describe the clinical, diagnosis and treatment of a complex renal mass and its histological confirmation after surgery. Review of the literature.

Conclusions: kidney's hydatidose is an unusual placement of this pathology. It is important to take care in the differential diagnosis in the context of complexes renal masses. There are some diagnosis procedures which can help us to establish it. Surgery is the treatment of choice in the majority of the cases.

Keywords: Hydatid cyst of the kidney.

$\mathrm{L}^{\mathrm{a}}$ a hidatidosis ó equinococosis es una parasitosis zoonótica causada por céstodos del género Echinococcus.

La enfermedad hidatídica es un problema de salud importante en países en vías de desarrollo; característicamente endémica en zonas de crianza de ganado ovino. En nuestro entorno es una patología poco frecuente ${ }^{1}$.

La afectación renal es poco común, incluso en las poblaciones más afectadas. Se calcula que representa entre el 1-4\% de todos los quistes hidatídicos ${ }^{3-4}$.
A continuación presentamos un nuevo caso de quiste hidatídico renal, en el contexto de una masa quística compleja, cuyo diagnóstico se realizó tras el tratamiento quirúrgico.

\section{CASO CLÍNICO}

Mujer de 73 años de edad remitida a consultas para valoración de masa renal derecha. Como antecedentes personales destacan una diverticulosis de colon, la extirpación de un nódulo tiroideo benigno y la exéresis 15 años atrás de un quiste hidatídico pulmonar. 
A raíz de un cuadro de lumbalgia derecha de 1 mes de evolución se le practicó ecografía renovesical en la que se apreciaba una masa renal derecha sólida de $4 \mathrm{~cm}$. en polo inferior, altamente sugestiva de hipernefroma (Fig.1). No refirió episodios de hematuria ni otra sintomatología importante. La analítica sanguínea y la exploración física fueron estrictamente normales.

Se solicitó TAC abdomino-pélvico, que confirmó masa de aproximadamente $4 \mathrm{~cm}$. de diámetro, localizada en polo inferior de riñón derecho, sólidoquística y con calcificación parietal sin captación de contraste evidente (Fig. 2 y 3 ).

Con la orientación diagnóstica de quiste complicado vs hipernefroma se decidió intervención quirúrgica, practicándose nefrectomía parcial polar inferior derecha.

El estudio anatomopatológico informó de quiste hidatídico renal sin signos de actividad.

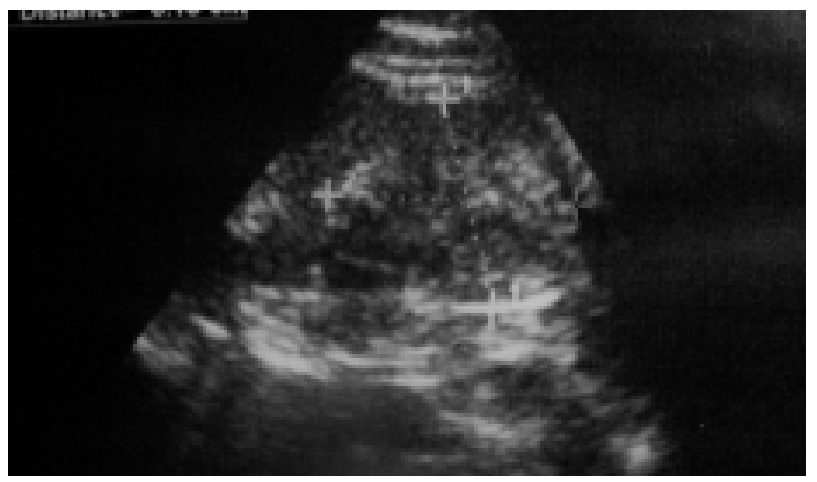

FIGURA 1. Ecografia renal: masa sólida de aproximadamente $4 \mathrm{~cm}$. en parénquima de polo inferior derecho altamente sugestivo de hipernefroma. No compromiso vascular.

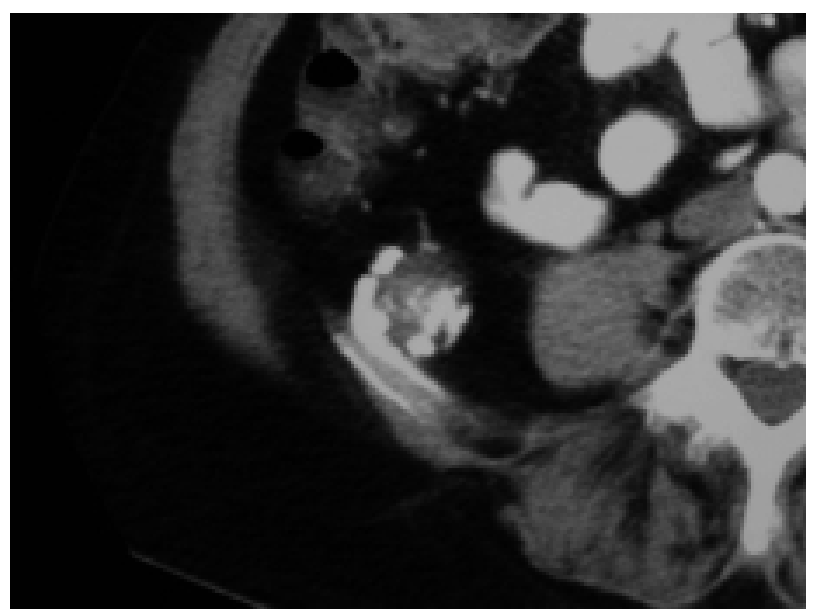

FIGURA 2. TAC: masa sólido-quística y con calcificación parietal.

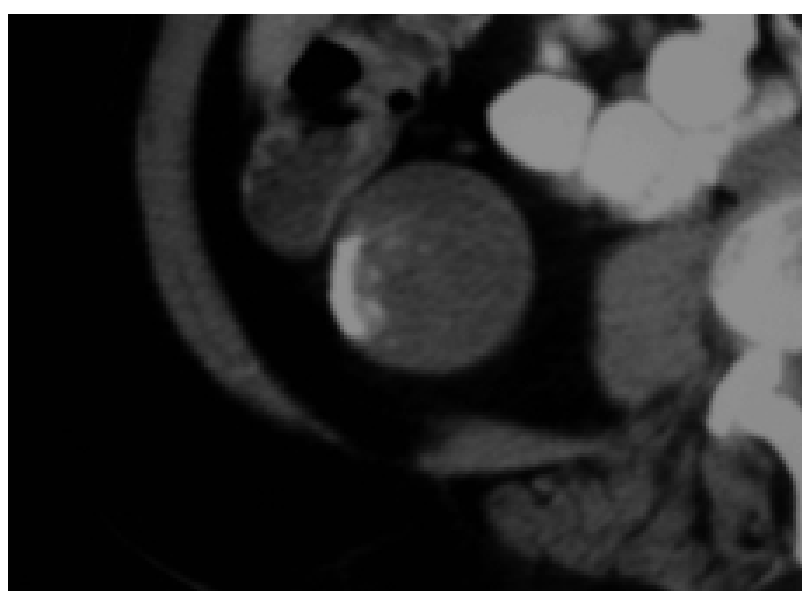

FIGURA 3. TAC: no existe captación evidente del contraste.

Tras 12 meses de seguimiento la paciente se encuentra asintomática y con buena función renal.

\section{DISCUSIÓN}

La hidatidosis o equinococosis es conocida desde tiempos de Hipócrates, pero no es hasta 1782 cuando Goeze descubrió la relación entre el gusano Echinococcus granulosus y la formación de quistes ${ }^{2}$.

Se trata de una zoonosis causada por céstodos del género Echinococcus.

Los humanos constituyen un huésped intermedio infrecuente, si bien los huéspedes habituales son las vacas, cerdos y ovejas, aunque el huésped definitivo es el perro, lobo y el zorro.

El ciclo se inicia tras la ingestión oral de los huevos que tras eclosionar en el intestino delgado dan lugar a las oncosferas o embriones hexacantos, que traspasan la pared intestinal y llegan a los vasos sanguíneos, alcanzando la vena porta y el hígado. En el $75 \%$ de los casos las oncosferas se fijan a éste; ocasionalmente atraviesan el hígado para llegar a los pulmones o más raramente al bazo, SNC, huesos, riñones o corazón. En cada uno de esos órganos las oncosferas forman quistes hidatídicos que se desarrollan durante meses o años creciendo lentamente. Los quistes hidatídicos están formados por una cápsula fibrosa (adventicia) que los separa del resto del parénquima sano. Internamente presentan una capa laminar (capa germinativa) de la cual se originan quistes secundarios o vesículas hijas; es a partir de éstos últimos donde se desarrollan las larvas (proscólex), que tras su maduración precipitan al fondo del quiste formando la llamada "arenilla hidatídica"2. 
Los huevos pueden llegar hasta los individuos que tienen un estrecho contacto con perros infectados; se encuentran en la saliva y las heces del perro, y la mayoría de perros infectados también tienen huevos de equinococos en el pelaje ${ }^{2}$.

En la mayor serie recogida de quistes hidatídicos (14.000 casos) el riñón representa el 1\% (hígado, 52\%; pulmón 36\%) ${ }^{3}$. Se puede encontrar a cualquier edad aunque es infrecuente en la infancia. Es más frecuente en mujeres ${ }^{3}$.

No existe una clínica específica. La hidatiduria, caracterizada como la presencia en la orina de material gelatinoso y de membranas que recuerdan "pellejos de uva", es un signo patognomónico de la presencia de un quiste hidatídico que ha drenado hacia la vía urinaria ${ }^{3-4}$.

La serología es una herramienta útil en el diagnóstico. La sensibilidad de los test serológicos varía en función de la localización de la enfermedad hidatídica ${ }^{4}$. Así los quistes pulmonares, de bazo y riñón, se asocian con bajos niveles de anticuerpos ${ }^{4}$. Actualmente no existe un test serológico con una sensibilidad y especifidad del $100 \%$ para la detección de anticuerpos anti-E.granulosus ${ }^{6}$. Se pueden dar falsos positivos debido a reacciones cruzadas con otros parásitos como el Schistosoma ${ }^{5}$. Es por ello por lo que el resultado serológico ha de confrontarse con los hallazgos radiológicos.

La UIV puede mostrar un efecto masa o calcificación que distorsione los cálices renales ${ }^{7}$, si bien actualmente no constituye una exploración de primera línea.

La ecografía nos permite una mayor caracterización de las masas renales quísticas. Existe una clasificación ecográfica de los quistes según su morfología, estructura y evolución, propuesta por Gharbi y coll $^{11}$. El tipo I es el quiste simple; en el II se ven finas paredes; en el III se demuestran septos evidentes; en el IV se objetiva material heterogéneo en su interior; el $\mathrm{V}$ corresponde a gruesas calcificaciones.

El TAC nos ayuda a hacer el diagnóstico diferencial con otras lesiones y asimismo, valorar otras localizaciones de la enfermedad hidatídica. Es importante intentar definir el carácter benigno o maligno de la lesión de cara al tratamiento definitivo. En el caso que presentamos existía la sospecha de que nos encontrábamos ante un hipernefroma por los hallazgos de la ecografía (consis- tencia sólida), pero el escáner demostraba una lesión sólido-quística sin captación evidente de contraste que sugería más una lesión benigna.

El tratamiento es quirúrgico para evitar la rotura de los quistes, y así su diseminación y/ó una reacción anafiláctica grave ${ }^{10}$. Previamente a la cirugía se pueden administrar distintos agentes para prevenir una posible abertura accidental durante el acto quirúrgico (formalina 10\%, nitrato de plata $0,5 \%$, albendazol, etc) ${ }^{8}$. Si no es posible la cirugía, puede realizarse un tratamiento conservador con albendazol, mebendazol o pracicuantel, pero la tasa de éxito es inferior al $20 \%$ en todas las series ${ }^{3}$.

Algunos grupos han preconizado la punción percutánea de los quistes, a pesar de los riesgos que ésto conlleva. Para prevenir las complicaciones se recomienda la administración preoperatoria de albendazol junto a un antihistamínico ${ }^{9}$.

\section{REFERENCIAS}

1. Abi F, el Fares F, Khaiz D, Bouzidi A. Les localisations inhabituelles du kyste hydatique: A propos de 40 cas. J Chir 1989;126(5):307-312

2. Buckley RJ, Smith S, Herschorn S, Comisarow RH, Barkin M..Echinococcal disease of the kidney presenting as a renal filling defect. J Urol 198;133:660-661.

3. El Idrissi AI, Mahjour $\mathrm{J}$ et Al. Retrospective survey for surgical cases of cystic echinococcosis in Morocco (1980-1992). Compendium on Cystic Ecchinococcosis in Africa and Middle Eastern Countries. Provo, Brigham Young University,1997:194206.

4. Craig PS. Immunodiagnosis of echinococcosis granulosus and comparaison of techniques for diagnosis of canine echinococcosis. Compendium on Cystic Echinococcosis in Africa and Middle Eastern Countries. Provo, Brigham Young University, 1997:85-118.

5. Pawlowski ZS. Critical points in the clinical management of cystic echinococcosis: a revised review. Compendium on Cystic Echinococcosis in Africa and Middle Eastern Countries. Provo, Brigham Young University, 1997:119-138.

6. Karabekios S, Gouliamos A, Kalovidouris A, Vlahos L, Papavasiliou C, Sakkas J Features of computed tomography in hydatid cysts of the urinary tract.. Br J Urol.1989;64(6):575-578.

7. Leemans J, Kotowicz A. Traitement chirurgical conservateur d'un kyste hydatique du rein. Acta Urol Belg 1975 Jul;43(3):257-262.

8. Saimot AG, Meulemans A, Cremieux AC, Giovanangeli MD, Hay JM, Delaitre B, Coulaud JP et al. Albendazole as a potential treatment for human hydatidosis. Lancet 1983; 2: 652-656.

9. Roylance J, Davies ER, Alexander WD.. Translumbar puncture of a renal hydatid cyst. Br J Radiol 1973; 46: 960-963.

10. Horchani A, Nouira Y, Kbaier I, Attyaoui F, Zribi AS. Hydatid cyst of the kydney: a report of 147 controlled cases. Eur Urol 2000; 38: 461-467.

11. Gharbi HA, Hassine W, Brauner MW, Dupuch K.. Ultrasound examination of the hydatic liver.Radiology. 1981;139(2):459-463.

Dr. J.Ma Abascal Junquera

Hospital Vall d’Hebron.Servicio de Urología

Passeig Vall d’Hebron 119-129 - Barcelona

josuvargas@hotmail.com

(Trabajo recibido el 11 mayo de 2004) 